\title{
Stochastic buckling of self-assembled colloidal structures
}

\author{
Simon Stuij, ${ }^{1}$ Jan Maarten van Doorn, ${ }^{2}$ Thomas Kodger, ${ }^{2}$ Joris Sprakel, ${ }^{2}$ Corentin Coulais, ${ }^{1}$ and Peter Schall ${ }^{1}$ \\ ${ }^{1}$ Institute of Physics, University of Amsterdam, Science Park 904, 1098 XH Amsterdam, Netherlands \\ ${ }^{2}$ Physical Chemistry and Soft Matter, Wageningen University, Stippeneng 4, 6708 WE Wageningen, Netherlands
}

(Received 19 January 2019; revised manuscript received 10 June 2019; published 27 September 2019)

\begin{abstract}
The vast majority of soft and biological materials, gels, and tissues are made from micrometer-size slender structures such as biofilaments and colloidal and molecular chains, which are believed to crucially control their mechanics. These constituents show intriguing extreme mechanics, mechanical instabilities, and plasticity, which, besides attracting significant theoretical attention, have not been studied experimentally and as such remain poorly understood. Here we investigate, by experiments, simulations, and theory, the mechanical instabilities of a slender self-assembled colloidal structure, observing a form of stochastic buckling where thermal fluctuations and associated entropic force effects are amplified in the vicinity of a buckling instability. We fully characterize how the persistence length and plasticity control the stochastic buckling transition, leading to intriguing higher-order buckling modes. These results elucidate the interplay of geometrical, thermal, and plastic interactions in the nonlinear mechanics of thermal self-assembled structures, crucial to the mechanical response and function of fiber-based soft and biological materials, as well as the rational design of micro- and nanoscale architectures.
\end{abstract}

DOI: 10.1103/PhysRevResearch.1.023033

\section{INTRODUCTION}

Due to recent advances in colloidal synthesis and interaction control, colloidal self-assembly has become a promising platform for designer materials with controlled internal architecture and tunable physical properties [1-3], such as unprecedented photonic [4], shape-changing [5,6], and mechanical properties [7]. Self-assembled colloidal structures also form excellent model systems to describe complex and biological materials, such as gels [8,9], biological cell membranes [10], and filaments [11,12], or flocking behavior [13]. To date, there has been an extensive focus on the dynamical and structural aspects of self-assembly $[14,15]$, while the mechanical instabilities of self-assembled objects have been experimentally much less explored; yet they play a crucial role in the response of soft materials [16-20] from biological networks [21] to mechanical metamaterials [22]. Semiflexible biofilaments, polymers, and biological shells have been shown to undergo signatures of mechanical instabilities [23,24], on which thermal excitations can have an important effect [25-29]. However, experimental insight into these instabilities in synthetic architectures such as colloidal assemblies is lacking. In particular, potentially crucial factors such as the effective elastic interactions, the role of geometric nonlinearities, stochastic noise, and plasticity are virtually unexplored.

Here we focus on the simplest and most widespread form of a mechanical instability on the simplest form of a

Published by the American Physical Society under the terms of the Creative Commons Attribution 4.0 International license. Further distribution of this work must maintain attribution to the author(s) and the published article's title, journal citation, and DOI. self-assembled structure: the buckling of an initially straight colloidal chain upon compression. Combining optical tweezers and microscopy experiments, molecular dynamics simulations, and theory, we observe that such a thermal chain undergoes an elastic buckling instability upon compression, accompanied by divergence of thermal bending fluctuations. Molecular dynamics simulations and continuum modeling allow identification of the critical exponents and exploration of entropic effects in the full range of persistence length, from stiff to the freely jointed chain. Finally, we show that plastic rearrangements lead to localized deformation at higher compression that can lead to buckling into higher-order modes. These results, uncovering the nature of mechanical instabilities in self-assembled structures, provide a crucial step towards understanding the complex mechanics of soft architectures, central to the mechanical function of biological materials and the design of functional colloidal materials.

\section{METHODS}

\section{A. Experiments}

Our system consists of copolymer particles [30] that we assemble into chains using temperature-dependent critical Casimir attractions [31,32]. The attractive force arises from the confinement of fluctuations of a binary solvent between the surfaces of the colloidal particles. The particles have a radius of $r=1.25 \mu \mathrm{m}$ and are suspended in a binary solvent of lutidine and water with a lutidine weight fraction $c_{L}=0.32$ and with $5 \mathrm{mM}$ potassium chloride, in which they sediment into a quasi-two-dimensional layer. Salt $(5 \mathrm{mM}$ potassium chloride) is added to screen the electrostatic repulsion. By setting the temperature to $\Delta T=5.5^{\circ} \mathrm{C}$ below the critical temperature $T_{c}=33.6^{\circ} \mathrm{C}$, we induce an attraction with potential depth $E \sim 10 k_{B} T$ and range $\sim 0.01 r$ that causes assembly of 
the particles. We use optical tweezers to grab the ends of assembled colloidal chains.

For the optical tweezers, laser light of $1064 \mathrm{~nm}$ was used at a power of $20 \pm 5 \mathrm{~mW}$. The trap constants were determined by tracking the Brownian movement of a single colloidal particle in the trap and fitting its displacements from the trap center with a Gaussian distribution to obtain the standard deviation $\sigma_{\text {trap }}$. We used a long measurement time such that the out-of-trap displacements become Boltzmann distributed. Assuming a harmonic trap, the trap constant is then determined by $k=k_{B} T / \sigma_{\text {trap }}^{2}$. We obtain $k=0.9 \pm 0.2 \mathrm{pN} / \mu \mathrm{m}$, where the error is estimated based on the locating accuracy of $\epsilon_{\text {track }}=0.02 \mu \mathrm{m}$. The partial absorption of the laser light by the binary solvent causes a local heating of $0.5 \mathrm{~K}$ at the trap. This was determined by measuring the temperature at which phase separation occurs in the laser focus and subtracting that from the phase separation temperature when the laser is turned off. This temperature increase is expected to cause a slight increase of the critical Casimir attraction close to the trapped bead.

We used two optical tweezers to push on the colloidal chain as follows. Starting from a straight chain, we apply a compressive displacement $u$ by moving one of the optical tweezers at a constant rate of $27 \mathrm{~nm} / \mathrm{s}$ towards the other. We then image the individual particles at a frame rate of $20 \mathrm{~s}^{-1}$ and locate their centers in the image plane with an accuracy of $20 \mathrm{~nm}$ using particle-tracking software [33]. In addition, we measure the force exerted on the chain from the bead displacement out of the static trap using $F=k\left(y-y_{\text {trap }}\right)$, where $y$ and $y_{\text {trap }}$ are the positions of the trapped bead and trap center, respectively. We define $L$ as the end-to-end distance of the chain and $L_{0}=24.7 \pm 0.1 \mu \mathrm{m}$ as the end-to-end distance for vanishing force $F=0$.

\section{B. Molecular dynamics simulations}

We support our experiments with molecular dynamics simulations of the buckling of a colloidal chain. Colloidal particles with position $\boldsymbol{r}_{\boldsymbol{i}}$ in an assembled chain satisfy the Langevin equation

$$
m \ddot{\boldsymbol{r}_{i}}=-\frac{k_{B} T}{D} \dot{\boldsymbol{r}}-\nabla_{\mathbf{r}_{i}} V+\sqrt{2 D} \xi
$$

with $D=k T / \gamma$ the diffusion coefficient, $\gamma$ the viscous drag coefficient, $\xi$ a normalized stochastic force, and

$$
V=\frac{k}{2} d_{0}^{2} \sum_{i=1}^{N-1}\left(\epsilon_{i}-1\right)^{2}+\frac{k_{\theta}}{2} \sum_{i=1}^{N-2}\left(\theta_{i}-\theta_{i, 0}\right)^{2},
$$

where $d_{0}$ is the equilibrium bond distance, $\theta_{i, 0}$ is the equilibrium angle, $\epsilon_{i}=\left|\boldsymbol{r}_{i+1}-\boldsymbol{r}_{i}\right| / d_{0}-1$, and $\cos \left(\theta_{i}\right)=\left(\boldsymbol{r}_{i+2}-\right.$ $\left.\boldsymbol{r}_{i+1}\right) \cdot\left(\boldsymbol{r}_{i+1}-\boldsymbol{r}_{i}\right) /\left|\boldsymbol{r}_{i+2}-\boldsymbol{r}_{i+1}\right|\left|\boldsymbol{r}_{i+1}-\boldsymbol{r}_{i}\right|$. On timescales $\Delta t>$ $\frac{m D}{k_{B} T}$ the Langevin equation can be considered overdamped and reduces to

$$
\dot{\boldsymbol{r}}_{i}=-\frac{D}{k_{B} T} \nabla_{\mathbf{r}_{i}} V+\sqrt{2 D} \xi
$$

These can be simulated by molecular dynamics (MD) simulations following the Ermak-McCammon equation [31]

$$
\boldsymbol{r}_{i}(t+\Delta t)-\boldsymbol{r}_{i}(t)=-\frac{D}{k_{B} T} \nabla_{\mathbf{r}_{i}} V \Delta t+\sqrt{2 D \Delta t} \xi .
$$

We simulate an infinitely stiff trap by fixing the positions of the two end particles. Trap movement is then implemented by moving one end particle towards the other.

Time, length, and energy are expressed in natural units such that $t_{D}=d_{0}^{2} / D=1, k_{B} T=1$, and $d_{0}=1$. In all simulations the time step was set to $\Delta t=2^{-16} t_{D}$, which is small enough to have a stable integration. In order to compare with experiments, all quantities were later rescaled using the experimental values $D=0.138 \mu \mathrm{m}^{2} / \mathrm{s}$ and $k_{B} T=4.14 \times 10^{-21} \mathrm{~J}$ corresponding to $\sim 0.004 \mathrm{pN} \mu \mathrm{m}$, and $d_{0}=2.74 \mu \mathrm{m}$.

The experimental values of the bending and stretching stiffness as determined from the experimental force are, respectively, $k_{\theta}=1048 k_{B} T$ and $k=14760 k_{B} T / d_{0}^{2}$. To simulate the compression experiment, we moved the trap in 128 steps of $\Delta u=0.01 d_{0}$, starting from $u_{i}=-0.3 d_{0}$, with a waiting time of $t_{\text {step }}=32 t_{D}$ at each step. This gives a total displacement similar to that from the experiment. The compression speed is much lower than in the experiments in order to obtain better statistics. For the analysis, we disregarded the first $8 t_{D}$ after each trap displacement to allow for equilibration. In experimental units, this entire ramp translates to a total displacement of $u_{\mathrm{tot}}=3.5 \mu \mathrm{m}$ over a time of $62 \mathrm{~h}$ with each step taking $30 \mathrm{~min}$, giving an effective speed of $v_{\text {trap }}=$ $0.9 \mathrm{~nm} / \mathrm{min}$.

We further extended the simulations by incorporating elastoplastic effects. In these simulations, we allowed an instant plastic relaxation to occur at a threshold angle $\theta_{p}$ such that the new equilibrium bond angle becomes $\theta_{0, i}=\theta_{c}$. We used the same $k_{\theta}$ and $k$ values as for the previous elastic simulations. A continuously increasing trap displacement was simulated for a total time of $t_{\text {tot }}=1.9 t_{D}$, increasing from $u_{i}=-0.3 d_{0}$ to $u_{f}=2.5 d_{0}$. This translates to a trap speed of $v_{\text {trap }}=37 \mathrm{~nm} / \mathrm{s}$, close to the actual experimental value. In order to estimate the critical bending angle, we performed 50 simulation runs at a number of $\theta_{c}$ ranging from 0.1 to $0.2 \mathrm{rad}$, in steps of $0.01 \mathrm{rad}$. The best fitting $\theta_{c}$ was determined by comparing the average plastic compression $u_{p}$ to the experimental value. This gave $\theta_{c}=0.21 \mathrm{rad}$.

\section{RESULTS AND DISCUSSION}

\section{A. Euler buckling}

To investigate its buckling behavior, we subject the initially straight colloidal chain to continuously increasing compression. We observe that the chain undergoes a sharp buckling transition at a well-defined compressive displacement $u_{c}$, as shown in Fig. 1(b). In the vicinity of $u_{c}$, fluctuations significantly increase, in agreement with recent predictions [34], as clearly visible in the superposition of three reconstructed images in Fig. 1(c). Upon further compression, the fluctuations decrease again and finally a kink appears at a well-defined large compressive displacement $u_{p}$.

To further elucidate this buckling behavior, we measure the force exerted by the trap on the chain as a function of the compressive displacement $u$ [Fig. 2(a)]. We observe a linear increase up to a critical displacement $u_{c}$ beyond which the force remains essentially constant. Such a forcedisplacement curve is strongly reminiscent of a classical Euler buckling problem [35-37]. To confirm the validity of this 


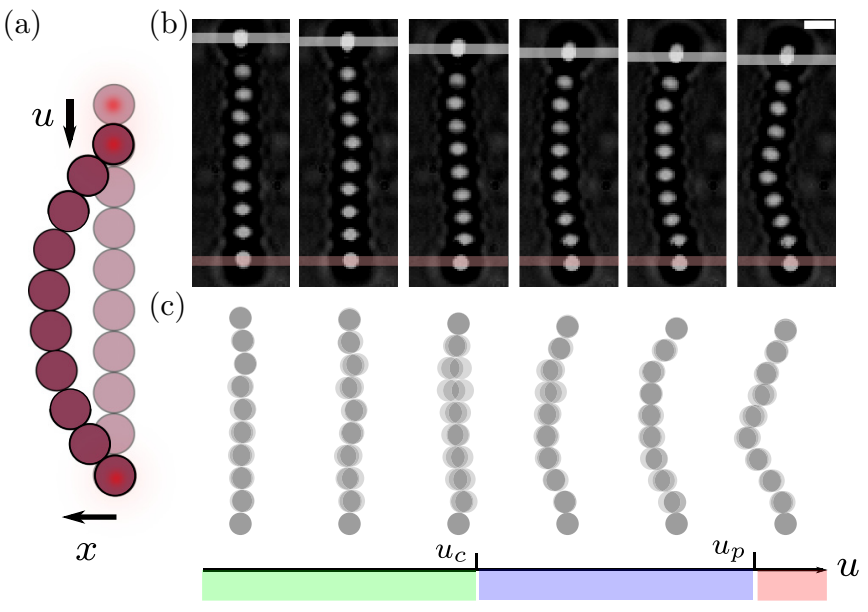

FIG. 1. Buckling of a colloidal chain. (a) Sketch of the colloidal chain compressed by optical tweezers (red dots). (b) Bright-field microscope images of the chain at $u=-0.45,-0.25,0.2,0.6,0.8$, and $1.0 \mu \mathrm{m}$, respectively from left to right, under a compressive displacement. White bars indicate the position of the laser trap that is slowly displaced and red bars the position of the static trap. The scale bar is $3 \mu \mathrm{m}$. The color code demarcates regions of the straight and elastically and plastically buckled chains, bounded by $u_{c}$ and $u_{p}$, respectively. (c) Overlay of three reconstructed chains, one corresponding to the still shown in (b), one taken $1.5 \mathrm{~s}$ earlier (light gray) and one $1.5 \mathrm{~s}$ later (dark gray). (See Supplemental video 1 [38] for microscopy video.)

analogy, we map our result onto that of a continuous beam. We use the Euler buckling criterion for the critical force $F_{c}=\pi^{2} B / L_{0}^{2}$ and the critical displacement $u_{c}=F_{c} / S$, where $B$ is the bending modulus and $S$ the linear stiffness of the beam. Determining the critical force $F_{c}=0.19 \pm 0.02 \mathrm{pN}$ and displacement $u_{c}=0.21 \pm 0.02 \mu \mathrm{m}$ by interpolation, we find that the bending rigidity of the chain is $B=11.9 \pm$ $1 \mathrm{pN} \mu \mathrm{m}^{2}$ and the linear stiffness is $S=0.9 \pm 0.1 \mathrm{pN} / \mu \mathrm{m}$, corresponding to a persistence length of $L_{p}=B / k_{B} T=$ $2900 \pm 240 \mu \mathrm{m}$, similar to microtubules [21]. Furthermore, this stiffness value is consistent with that obtained from a linear fit to the prebuckling slope $S=0.8 \pm 0.1 \mathrm{pN} / \mu \mathrm{m}$. Such excellent agreement between a model for athermal slender structures and our thermally activated colloidal chain is striking.

The validity of this mapping is further confirmed by the shape of the buckled state, which we quantify by the amplitude $M_{1}$ of the first Fourier mode of the beam deflection (see the Appendix for details) as a function of the compressive displacement $u$ [Fig. 2(b)]. While this amplitude is close to zero in the prebuckling regime $u<u_{c}$, it sharply departs from zero and increases as $M_{1} \propto\left(u-u_{c}\right)^{1 / 2}$ beyond the buckling point [see Fig. 2(b) inset]. Again, this result is qualitatively similar to that of a macroscopic Euler buckling problem [35-37]. Note that such a deflection-displacement curve provides an independent measure of the critical displacement $u_{c}=0.21 \pm 0.02 \mu \mathrm{m}$, which is equal to the previous one within experimental errors. These results are consistent with and rationalize previous studies reporting a bending rigidity of linear assembled structures [39-41].

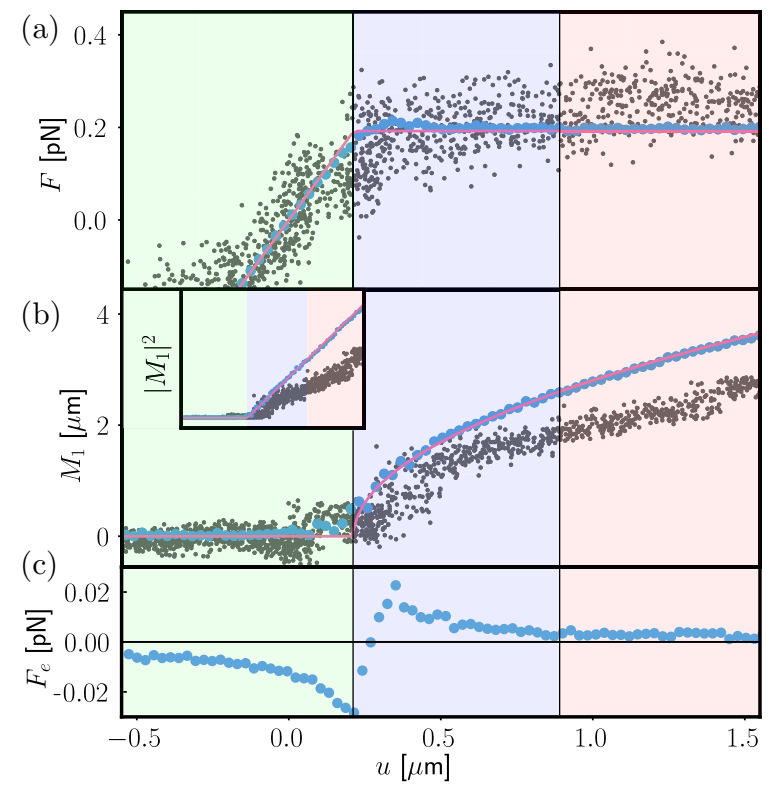

FIG. 2. Elastic buckling regime. The bending force and first Fourier mode are from experiments (gray dots), simulations (blue dots), and the continuum model (purple line). (a) Compressive force $F$ exerted by the tweezers on the chain versus displacement $u$. Note that all experimental data points are depicted, while the simulation data have been averaged over fixed $u$. (b) Amplitude of the first Fourier mode $M_{1}$ of the particle deflections. Only positive mode amplitudes corresponding to positive deflection of the chain are shown. The inset shows the same quantity squared. (c) Entropic force from simulations (see the text).

\section{B. Numerical simulations}

We further rationalize the experimental findings by molecular dynamics simulations of elastically coupled particles in two dimensions subjected to thermal fluctuations (see Fig. 3). Specifically, we solve the overdamped Langevin equation [42]

$$
\dot{\boldsymbol{r}}_{i}=-\frac{D}{k_{B} T} \nabla_{\mathbf{r}_{i}} V+\sqrt{2 D} \xi,
$$

where $\xi$ is a normalized stochastic thermal force, $D=$ $0.138 \pm 0.1 \mu \mathrm{m}^{2} / \mathrm{s}$ is the diffusion coefficient measured experimentally by tracking diffusing colloids, and $T$ is the temperature equal to the experimental temperature. The potential energy is given by

$$
V=\frac{k}{2} d_{0}^{2} \sum_{i=1}^{N-1} \epsilon_{i}^{2}+\frac{k_{\theta}}{2} \sum_{i=1}^{N-2}\left(\theta_{i}-\theta_{i, 0}\right)^{2},
$$

with $\epsilon_{i}$ the extension of bond $i, \theta_{i}$ the angle between bonds $i$ and $i+1$, and $\theta_{i, 0}$ the equilibrium angles which vanish for the initially straight chain. The equilibrium bond distance is determined from experiments as the average particles separation
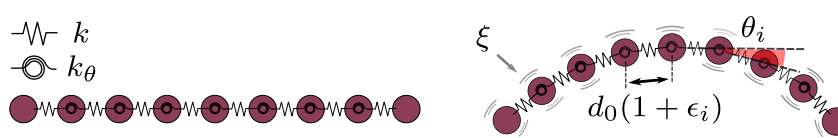

FIG. 3. Schematic of the model system used for the simulations and the analytical model. 
$d_{0}=L_{0} / N-1$. We also take the bending rigidity and bond stiffness from the experimental measurements $k=S(N-1)$ and $k_{\theta}=B / d_{0}$ and assume an infinite trapping potential. We then apply compression by moving the traps stepwise towards each other with a displacement $u_{\text {step }}=0.01 d_{0}$ and waiting time $t_{\text {step }}=32 d_{0}^{2} / D$ between each step, yielding an average compression rate of $0.9 \mathrm{~nm} / \mathrm{min}$, much slower than the experiments, allowing us to acquire good statistics. Despite the simple assumptions of the numerical model, the results are in good agreement with the experiments (see Fig. 2). The force and deflection curves both predict the buckling instability at $u_{c}$ and correctly describe the force behavior; the quantitative deviations are likely due to (i) plastic effects and (ii) the fact that the experimental boundary conditions (laser traps) do not allow completely free rotations of the trapped colloids.

We can use the simulations to extract the entropic contribution $F_{e}$ to the compressive force associated with the thermal fluctuations of the colloidal chain. We do so by subtracting from the compressive force at room temperature the force at zero temperature. In Fig. 2(c) $F_{e}$ shows a characteristic signature of the buckling transition: It diverges and changes sign at the buckling transition (from compressive to repulsive), reflecting the change in the contour length of the chain upon buckling and the associated change in the number of chain configurations. However, the magnitude of $F_{e}$ is just below the experimental resolution and cannot be resolved experimentally.

\section{Fluctuations}

Nevertheless, we can measure the fluctuations directly by monitoring the variance of the Fourier amplitudes. We focus on the first mode and compute its variance $\sigma_{M_{1}}^{2}=$ $\left\langle\left(M_{1}-\left|\bar{M}_{1}\right|\right)^{2}\right\rangle_{M_{1} \geqslant\left|\bar{M}_{1}\right|}$. Upon approaching the buckling point, this variance grows and diverges [Fig. 4(a)]. The doublelogarithmic plot (inset) suggests a divergence $\sigma_{M_{1}}^{2} \sim \mid u-$ $\left.u_{c}\right|^{-v}$ with exponent $v=1$. We also measure the typical timescale of fluctuations $\tau_{M_{1}}$ from exponential fits to the decay of the autocorrelation function $C(\Delta t)=\left\langle M_{1}(t) M_{1}(t+\Delta t)\right\rangle$; this fluctuation time shows likewise a significant increase upon approaching $u_{c}$ [see Fig. 4(b)]. However, the uncertainty and limited number of data points do not allow us to pinpoint the divergence of these growing fluctuations quantitatively.

\section{Continuum model}

This stochastic buckling transition is described in a simple (analytically solvable) continuum limit of Eq. (2), known as the extensible elastica [43]. In this limit, the energy can be decomposed into independent contributions from each Fourier mode. To first order in $u$, the energy dependence on the first mode amplitude $M_{1}$ becomes a double well, given by

$$
V_{1}=\frac{S \pi^{2}}{4 L_{0}}\left(u_{c}-u\right) M_{1}^{2}+\frac{S \pi^{4}}{32 L_{0}^{2}} M_{1}^{4}+\mathcal{O}\left(u^{2}\right)+\mathcal{O}\left(M_{1}^{6}\right)
$$

where $u_{c}=B \pi^{2} / S L_{0}^{2}, B$ is the bending rigidity, and $S$ is the stretching stiffness. Higher modes exhibit a single harmonic energy dependence and equilibrate to zero (see the Appendix). Mechanical equilibria of this extensible elastica, prescribed by the condition $\partial V_{1} / \partial M_{1}=0$, are given by $M_{1, m}=0$ in the prebuckling regime $\left(u<u_{c}\right)$ and by $M_{1, m}= \pm 2 / \pi \sqrt{L_{0}\left(u-u_{c}\right)}$ in the postbuckling regime $\left(u>u_{c}\right)$. The corresponding forces
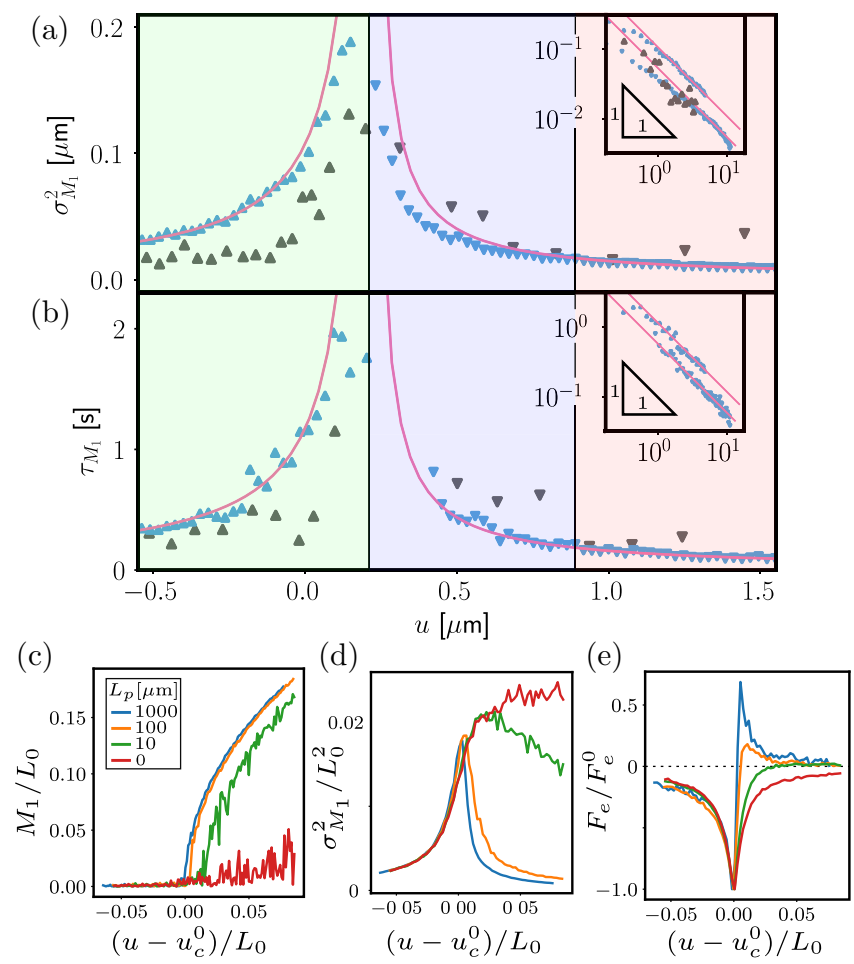

FIG. 4. Fluctuations close to buckling. (a) Variance $\sigma_{M_{1}}^{2}$ of $M_{1}$ above the mode of its distribution versus the compressive displacement $u$. The inset shows a log-log plot of $\sigma_{M_{1}}^{2}$ versus $\left|u-u_{c}\right| / u$. (b) Correlation time $\tau_{M_{1}}$ of $M_{1}$. The inset shows a log-log plot of $\tau_{M_{1}}$ versus $\left|u-u_{c}\right| / u$. Experimental, numerical, and continuum model data are represented by gray triangles, blue triangles, and purple lines, respectively. Simulation and continuum model values are divided by a factor of 3 to fit on the same axis. Also shown are the MD simulations for decreasing chain persistence length showing (c) the normalized first Fourier amplitude $M_{1}$, (d) the variance, and (e) the entropic force $F_{e}$ versus normalized $u$ shifted by the zero-temperature buckling compression $u_{c}^{0}=\pi^{2} B / S L_{0}^{2}$. Blue, orange, green, and red curves correspond, respectively, to $1,1 / 10,1 / 100$, and 0 times the experimental bending rigidity. The variance was calculated over a limited time window of $1 \mathrm{~s}$. (See Supplemental video 2 [38] for an animation of the simulation data.)

are $F_{m}=k u / 2$ for $u<u_{c}$ and $F_{m}=F_{c}\left(1+\left(u-u_{c}\right) / 2 L_{0}\right)$ for $u>u_{c}$. Furthermore, assuming that bending energies obey a Boltzmann distribution in equilibrium, one finds that both $\sigma_{M_{1}}^{2}$ and $\tau_{M_{1}}$ diverge with a power of -1 (see the Appendix). These predictions are in perfect agreement with the experiments and simulations as shown in Figs. 2 and 4 (pink curves). A physically appealing picture emerges from these results: Once in the presence of stochastic noise, the classical buckling transition remains a supercritical bifurcation, but the vicinity of the bifurcation is associated with fluctuations of diverging magnitude and timescale.

This mean-field buckling behavior diminishes for higher fluctuations. We simulated chains with lower bending rigidities exhibiting stronger fluctuations and find that the buckling transition loses its sharp character and eventually vanishes [Fig. 4(c)]. Concomitantly, the divergence of buckling fluctuations broadens and eventually disappears [Fig. 4(d)]. This is associated with a striking change in the entropic force 


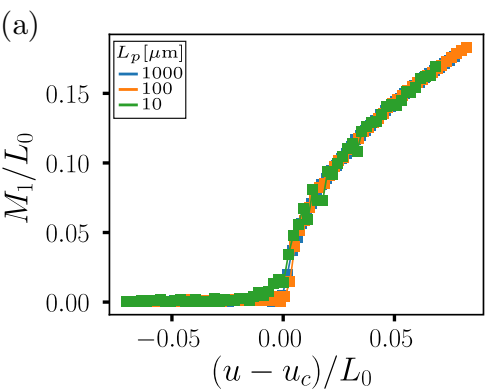

(b)

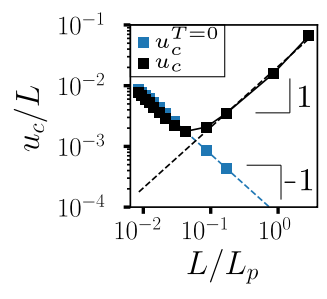

FIG. 5. Transition to low persistence length. (a) Scaling collapse of the first mode amplitude $M_{1}$ versus normalized $u-u_{c}$, with $u_{c}$ the buckling displacement. (See Supplemental video 3 [38] for an animation of the simulation data.) (b) Extracted normalized buckling point $u_{c}$ as a function of flexibility. Two scaling regimes are evident: exponent -1 associated with the chain compressibility for low flexibility (high bending stiffness) and a regime with exponent +1 associated with the loss of bending rigidity. (See Supplemental video 4 [38] for microscopy video.)

[Fig. 4(e)] that loses its characteristic change of sign: The positive branch at $\left|u-u_{c}^{0}\right|>0$ vanishes, indicating that the gradual transition to the freely jointed chain is always attracted to zero end-to-end distance. These results are in qualitative agreement with the analytic results for a filament with decreasing bending rigidity in Ref. [28]. Furthermore, other routes towards stronger fluctuations reveal a similar loss of the buckling transition as shown by simulations of longer chains and higher temperatures in the Appendix. To fully elucidate the transition from rigid to flexible chains, we collapse the buckling curves in Fig. 4(c) by plotting them versus $u-u_{c}$, where we subtract the critical buckling displacement [see Fig. 5(a)]. The extracted $u_{c}$ shows two scaling regimes: It first decreases and then increases with increasing chain flexibility [see Fig. 5(b)]. The initial decrease with exponent -1 is due to the additional compressive component of the chain; the same decrease is observed for vanishing temperature (blue data $u_{c}^{T=0}$ ), for which, according to Euler buckling, $u_{c}$ is given by $u_{c}^{T=0}=F_{c} / S \propto L_{p}^{-1}$, thus the exponent -1 . Towards higher flexibility, however, $u_{c}$ grows with exponent +1 , reflecting the transition from a rigid beam to a freely joint chain, for which $u_{c}$ becomes infinite. We thus find that the buckling transition increases linearly with increasing flexibility of the chain. Thus, it gradually diminishes as the energetic advantage of buckling in the lowest mode ceases together with the bending rigidity.

\section{E. Plastic buckling}

We also explored plastic effects. At even larger displacements $u>u_{p}$, the chain undergoes localized bending deformations as shown in Figs. 1(b) and 1(c) (rightmost images), which we find to be irreversible upon releasing the applied compression. To quantify this degree of localization, similar to plastic events in amorphous materials, we calculate the inverse participation ratio (IPR), which varies between $N-2$ for fully localized deformations and 1 for distributed deformations, as defined by

$$
\mathrm{IPR}=(N-2) \frac{\sum_{i=1}^{N-2} \hat{\theta}_{i}^{4}}{\left(\sum_{i=1}^{N-2} \hat{\theta}_{i}^{2}\right)^{2}}
$$
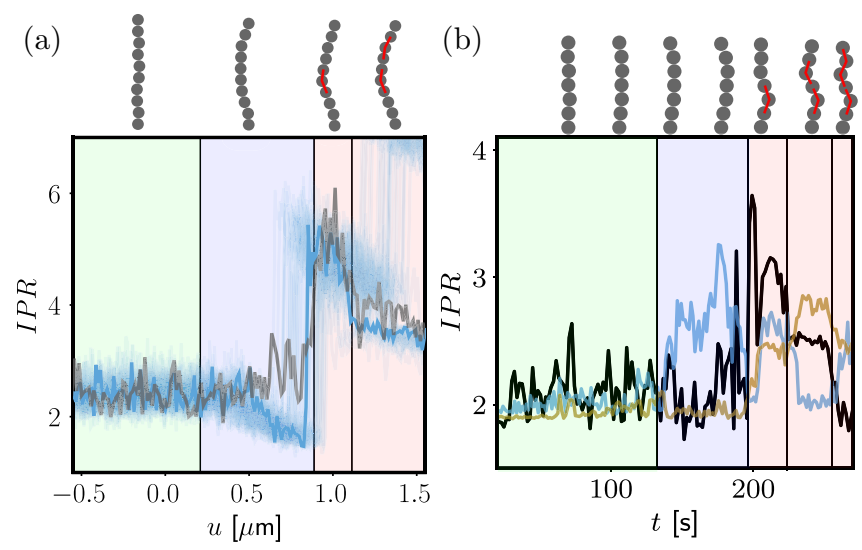

FIG. 6. Plastic buckling. (a) Inverse participation ratio of the experimental chain (gray) and of 50 independent MD simulation runs (blue shading) as a function of continuously increasing compression. The simulations are performed with elastic parameters as in Fig. 2 and $\theta_{c}=0.21 \mathrm{rad}$. Vertical lines and colors distinguish regimes of the straight and elastically and plastically buckled chains and indicate two plastic slippage events. Reconstructed snapshots show the experimental chain for $u=0,0.5,1$, and $1.5 \mu \mathrm{m}$, with snapped bonds highlighted in red. (b) The IPR (black), $M_{1}$ (blue), and $M_{2}$ (yellow) versus time of a different compression experiment. Here the chain was shorter $(N=7)$ and the trap was moved stepwise, by $\delta u=0.1 \mu \mathrm{m}$ every $60 \mathrm{~s}$.

Here $\hat{\theta}_{i}=\left|\theta_{i}\right|-\left\langle\left|\theta_{i}\right|\right\rangle_{u<u_{c}}$, i.e., the local angular deviation from the straight chain. When the chain buckles elastically, the IPR remains small [see the gray curve in Fig. 6(a)], while at larger compression $u=u_{p}=0.89 \pm 0.02 \mu \mathrm{m}$, when the chain develops a kink, a clear spike appears. The value of about 6 , which is only slightly smaller than the maximum $N-$ $2=8$, indeed suggests very localized deformations. These features can be easily reproduced in the simulations, when we augment our numerical model with a simple elastoplastic model. Beyond a threshold angle $\theta_{p}$, an instant plastic relaxation occurs such that the equilibrium bond angle becomes $\theta_{0, i}=\theta_{p}$. Taking a value $\theta_{p}=0.21 \mathrm{rad}$ gives results qualitatively and quantitatively similar to the experiment [see blue shading in Fig. 6(a)]. By repeating 50 simulations we obtain an average $u_{p \text {,sim }}=0.84 \pm 0.09 \mu \mathrm{m}$, which indeed corresponds to the value $u_{p}$ observed in the experiments. Intriguingly, the combination of elastoplastic dynamics and thermal noise can further lead to higher-order buckling modes when the chain is compressed at higher compression rates [Fig. 5(b)]. We observe a sequence of buckling transitions through mode 1 , mode 2 , and mode 3 , which we interpret as a sequence of plastic events, as clearly shown by the mode 1 and 2 amplitudes (blue and olive) and the IPR (black).

\section{SUMMARY AND OUTLOOK}

We have experimentally demonstrated the rich stochastic buckling dynamics for a colloidal chain under uniaxial compression and rationalized our experimental results by simulations and analytic modeling. In the elastic regime, bending interactions and stochastic noise lead to diverging bending fluctuations. With decreasing persistence length, as 
higher-order modes are excited, this divergence smoothens, and the buckling transition eventually vanishes. Important biological filaments have a persistence length of, e.g., $L_{p}=$ $5000 \mu \mathrm{m}$ for microtubules of similar order to our colloidal chain, while for actin filaments $L_{p}=17 \mu \mathrm{m}$, putting those in the large fluctuation regime where the buckling transition smoothens [44]. These results have important consequences for the mechanics of biological tissue [21], colloidal gels, and even granular force chains $[45,46]$. Depending on the mesh size relative to the persistence lengths, filament buckling interactions become important and the observed divergence of fluctuations then translates into an entropic contribution to the stress, which should manifest in the rheology of such networks. Our results open up avenues for self-assembled colloidal structures with advanced nonlinear mechanics of relevance for the understanding of the rheology of gels [9], the mechanics of living tissues [21], and designer colloidal architectures [22].

\section{ACKNOWLEDGMENTS}

C.C., T.K., J.S., and P.S. acknowledge support from, respectively, Veni, Veni, Vidi, and Vici Fellowships from the Netherlands Organization for Scientific Research (NWO). J.M.v.D. acknowledges funding from the Industrial Partnership Program "Hybrid Soft Materials" of Unilever and NWO.

\section{APPENDIX}

\section{Mode definition and effective mode diffusion constant}

After locating the particles in the chain, a mode decomposition is performed in the following manner. First, the outof-line deflection of every nontrapped particle $j$ is calculated as the perpendicular distance $x_{j}$ from the line connecting the two trapped particles at the ends. Next a scaled discrete sine transform of type 1 is performed on $x_{j}$ defined by

$$
M_{i}=\frac{2}{N-1} \sum_{j=1}^{N-2} x_{j} \sin \left(\frac{\pi}{N-1} j i\right), \quad i=1, \ldots, N-2 .
$$

Here the normalization has been chosen such that

$$
x_{j}=\sum_{i=1}^{N-2} M_{i} \sin \left(i j \frac{\pi}{N-1}\right), \quad j=0, \ldots, N-1 .
$$

Based on the overdamped Langevin equation of individual colloids [Eq. (1)], we can derive an equivalent dynamical equation in terms of modes, given by

$$
\dot{M}_{i}=-\frac{D_{M}}{k_{B} T} \nabla_{M_{i}} V+\sqrt{2 D_{M}} \xi .
$$

Here $D_{M}$ is an effective diffusion coefficient. This coefficient is equal for all modes and can be derived by inserting Eq. (A2) in Eq. (1). This gives $D_{M}=2 D /(N-1)$.

\section{Theoretical model}

We parametrize the shape of an extensible elastica by $\boldsymbol{r}(s)=(x(s), y(s))$, with $s$ running from 0 to $L_{0}$, the rest length. The compressive strain is defined as $\gamma(s)=$ $\sqrt{(d x / d s)^{2}+(d y / d s)^{2}}$ and the orientation angle as $\phi(s)=$ $\arctan (d y / d x)$. The energy functional of an elastica including elastic energy and work exerted by a compressive force $F$ is given by [32]

$$
\begin{aligned}
V= & \frac{B}{2} \int_{0}^{L_{0}}\left(\frac{d \phi}{d s}\right)^{2} d s+\frac{L_{0} S}{2} \int_{0}^{L_{0}}(\gamma-1)^{2} d s \\
& +F\left(\int_{0}^{L_{0}} \gamma \cos (\phi) d s-R\right) .
\end{aligned}
$$

Here $R=L_{0}-u$ is the end-to-end length, $B$ is the bending rigidity, and $S$ is the stretching stiffness of the elastica. Minimizing $V$ with respect to $\gamma$ and $F$, we find

$$
\gamma=1-\frac{F}{S L_{0}} \cos \phi, \quad F=S L_{0} \frac{\int_{0}^{L_{0}} \cos \phi d s-R}{\int_{0}^{L_{0}}(\cos \phi)^{2} d s} .
$$

Inserting these back into Eq. (A4), we obtain an energy $V_{\phi}$ purely as a function of the orientation angle, given by

$$
V_{\phi}=\frac{B}{2} \int_{0}^{L_{0}}\left(\frac{d \phi}{d s}\right)^{2} d s+\frac{S L_{0}}{2} \frac{\int_{0}^{L_{0}} \cos (\phi) d s-R}{\int_{0}^{L_{0}}(\cos \phi)^{2} d s} .
$$

This is the energy we will use to determine the equilibrium angles $\phi(s)$ and also the size of thermal fluctuations in $\phi$. Note that it is indeed correct to use $V_{\phi}$ to determine the equilibrium. However, using $V_{\phi}$ to determine the size of fluctuations disregards the effect of thermal fluctuations in $\gamma$ and $F$. These fluctuations are not uncoupled from fluctuations in $\phi$, as can be seen from Eq. (A4). However, we assume that these fluctuations have negligible influence.

After a Fourier transform assuming Neumann boundary conditions $\phi=\sum_{n=1}^{\infty} \alpha_{n} \cos \left(\frac{n \pi}{L_{0}} s\right), V_{\phi}$ decomposes into $V_{\phi}=$ $S u^{2} / 2+\sum V_{\alpha_{n}}$. It follows that up to a critical compression $u_{c}$ all modes equilibrate to zero. After $u_{c}$, the first mode becomes nonzero and the chain buckles, which can be seen from

$$
\begin{aligned}
V_{\alpha_{1}}= & \frac{S L_{0}}{4}\left(u_{c}^{0}+\frac{u^{2}}{L_{0}}-u\right) \alpha_{1}^{2} \\
& +\frac{S}{32}\left(L_{0}^{2}-\frac{7 u L_{0}}{2}-2 u^{2}\right) \alpha_{1}^{4}+\mathcal{O}\left(\alpha_{1}^{6}\right),
\end{aligned}
$$

where $u_{c}^{0}=\pi^{2} B / S L_{0}^{2}$. The buckling compression of the first mode is found by determining the root of the term in front of $\alpha_{1}^{2}$, giving $u_{c}=\frac{L_{0}}{2}\left(1-\sqrt{1-4 u_{c}^{0} / L_{0}}\right)$. In the regime that we probe experimentally, $u_{c} / L_{0}$ and $u / L_{0}$ are small numbers. Therefore, $u_{c} \approx u_{c}^{0}$, and lowest order terms dominate in $V_{\alpha_{1}}$, which reduces to

$$
V_{\alpha_{1}}=\frac{S L_{0}}{4}\left(u_{c}^{0}-u\right) \alpha_{1}^{2}+\frac{S L_{0}^{2}}{32} \alpha_{1}^{4}+\mathcal{O}\left(u_{c}^{2}\right)+\mathcal{O}\left(u^{2}\right)+\mathcal{O}\left(\alpha_{1}^{6}\right) \text {. }
$$

Minimizing this energy, we see that the equilibrium first mode is given by

$$
\alpha_{1, m}^{2}= \begin{cases}0, & u<u_{c} \\ \frac{4}{L_{0}} \Delta u+\mathcal{O}\left(\Delta u^{2}\right), & u>u_{c},\end{cases}
$$

with $\Delta u=u-u_{c}$.

To derive the compressive force up to first order in $\Delta u$, care has to be taken to solve $\alpha_{1, m}^{2}$ from Eq. (A6) one order higher 

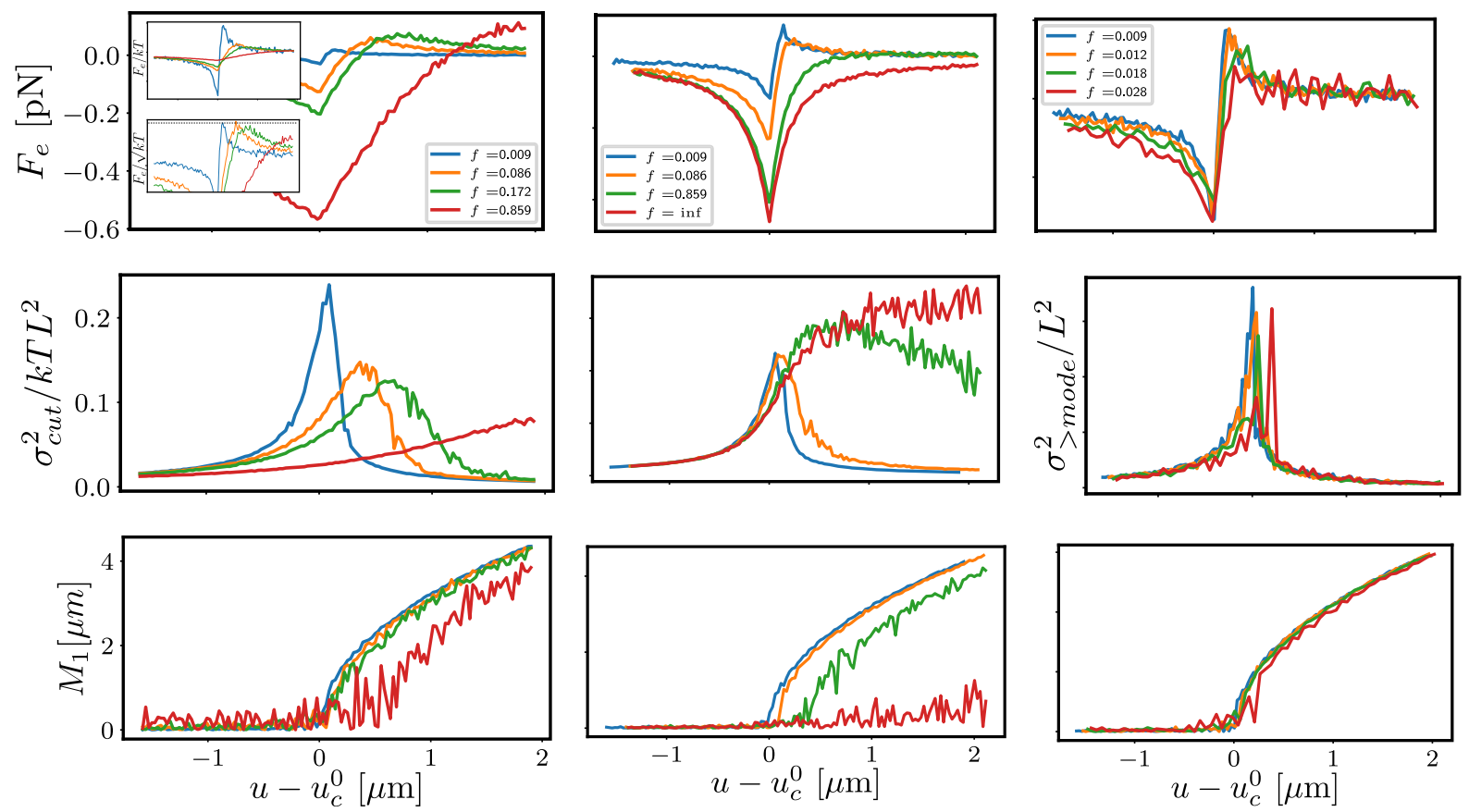

FIG. 7. MD simulation effect on $F_{e}$ and $\sigma^{2}$ of three different routes that increase the flexibility of the system: left column, increasing $T$; middle column, $B$; and right column $N$. Here $F_{e}$ is calculated by subtracting the nonthermal $F$ [Eq. (A9)] from the measured $F_{\text {sim }}, \sigma_{\text {cut }}^{2}$ is the variance of the $M_{1}$ over a time window of around $1 \mathrm{~s}$, and $\sigma_{>\text {mode }}^{2}$ are fluctuations above the mode of the distribution as defined in the main text. All simulations were run using the same protocol as described above for the elastic simulation.

in terms of $u_{c} / L_{0}$. Doing that and inserting in Eq. (A5), one obtains

$$
F= \begin{cases}S u, & u<u_{c} \\ F_{c}\left(1+\Delta u / 2 L_{0}\right)+\mathcal{O}\left(\Delta u^{2}\right), & u>u_{c}\end{cases}
$$

where $F_{c}=S u_{c}$.

As a last step to obtain the double-well potential stated in the main text we have to transform $\alpha_{1}$ to $M_{1}$, defined by Eq. (A1). Using that for small deflection and compressions, we have $M_{1}=L_{0} \alpha_{1} / \pi$, and Eq. (A7) becomes Eq. (3).

Furthermore, we can derive an expression for the variance $\sigma_{M_{1}}^{2}$ of the first-mode amplitude. If we assume that in equilibrium, the bending energies given by Eq. (A6) obey a Boltzmann distribution, then the mode fluctuations around the average become Gaussian distributed with variance

$$
\sigma_{M_{1}}^{2}= \begin{cases}\frac{2 k_{B} T L_{0}}{\pi^{2} S}\left|u_{c}-u\right|^{-1}, & u<u_{c} \\ \frac{k_{B} T L_{0}}{\pi^{2} S}\left|u_{c}-u\right|^{-1}, & u>u_{c} .\end{cases}
$$

Note that this approach breaks down for $u>\sim u_{c}$, in the postbuckling regime near the buckling point, where the distribution becomes bimodal rather than a single Gaussian as predicted by Eq. (A10). For the fluctuation time, the overdamped dynamics for a harmonic well predicts that $\tau_{M_{1}}=$ $\sigma_{M_{1}}^{2} / D_{M_{1}}$, where $D_{M_{1}}=2 D /(N-1)$ is the effective mode diffusion.

\section{Exploring the strong-fluctuation regime}

Here we investigate the large-fluctuation regime in more detail. Specifically, we address the effect of increasing fluctuations achieved by (i) increasing temperature, (ii) decreasing the bending rigidity, and (iii) increasing the number of particles of the colloidal chain in the MD simulations. Each of these routes increases the flexibility $f=L / L_{p}$ of the chain, where $L_{p}=B / k_{B} T$ is the persistence length and $L$ the total length of the chain. It has been predicted theoretically that for stiff to semiflexible compressible rods with persistence length $L_{p}<L$, thermal fluctuations contribute an additional entropic force $F_{e}$ [on top of the nonthermal compressive force $F$, Eq. (A9)] of $O(T)$ far away from $u_{c}$ and $O(\sqrt{T})$ near $u_{c}$ [34]. Other theoretical work, which assumes an incompressible semiflexible rod in two dimensions, predicts a critical entropic force increase $F_{e, c} \propto k_{B} T[27,28]$.

The simulation results for increasing temperature, decreasing bending rigidity, and increasing number of particles are shown in Fig. 7, columns from left to right, respectively. As a unique feature of the buckling transition, the entropic force (top row) switches sign when crossing $u_{c}$ from negative (favoring compression) to positive (favoring expansion). The negative (tensile) entropic force upon approaching $u_{c}$ from the left reflects the driving force towards larger number of configurations with decreasing end-to-end distance. At buckling, the force changes sign as the buckled state (mode 1 fluctuation) again suppresses the number of accessible configurations (higher-order fluctuations). The resulting positive (repulsive) entropic force leads to an extra buckling force barrier increasing the buckling compression; such an extra force barrier was predicted by the theoretical studies in Refs. [28,34]. Furthermore, we find, as predicted in Ref. [34] but contradicting [28], that for $L_{p}<L$ the amplitude of this positive peak goes as $O(\sqrt{T})$, as can be seen by the collapse of peak height when rescaling by $\sqrt{k_{B} T}$ (second inset top left). 
Further away from $u_{c}$ the entropic force scales with $O(T)$, as can be seen by the collapse when rescaling by $k_{B} T$ (first inset top left), also inline with [34]. The discrepancy between the two theoretical predictions, as well as our closer agreement with [34], could be explained by the fact that our simulation assumes a compressible chain, more closely agreeing with the theoretical model of [34].

The entropic effects clearly increase with increasing flexibility $f$ of the chain. As fluctuations become more prominent at higher temperature, for smaller bending stiffness, and for longer chains, the amplitude of the negative (prebuckling) entropic force grows. The negative region also extends as $u_{c}$ drifts to the right. This can be seen in the growing region in between the negative and positive peaks of $F_{e}$. The positive peak, on the other hand, decreases in amplitude (inset top left, top middle), until it vanishes and $F_{e}$ shows only attraction towards $u=0$, reflecting the behavior of a freely joint chain with a continuously increasing entropic tensile force upon decreasing end-to-end distance. These effects are less visible in the data of the increasing chain length (right column), which are limited to lower chain flexibilities due to computational costs of equilibrating long chains.

As a result of this trend, $u_{c}$ disappears: Very flexible chains lose the signatures of the buckling transition. This is most clearly observed in the gradual disappearance of the characteristic kink of the first-mode amplitude, as shown in the bottom row. At the same time, the divergence of fluctuations (middle row) decreases and eventually vanishes. Hence, the thermally activated buckling transition with its characteristic diverging fluctuations as described by mean-field theory is observed only in a limited range of small fluctuations, where the predominantly elastic chain (with flexibility $L / L_{p}<1$ ) has an energetic advantage of buckling into the lowest mode (opposed to the excitation of higher modes).
[1] V. N. Manoharan, Science 349, 1253751 (2015).

[2] D. Morphew and D. Chakrabarti, Curr. Opin. Colloid Interface Sci. 30, 70 (2017).

[3] Z. Gong, T. Hueckel, G. R. Yi, and S. Sacanna, Nature (London) 550, 234 (2017).

[4] J. F. Galisteo-López, M. Ibisate, R. Sapienza, L. S. FroufePérez, A. Blanco, and C. López, Adv. Mater. 23, 30 (2011).

[5] A. A. Shah, B. Schultz, W. Zhang, S. C. Glotzer, and M. J. Solomon, Nat. Mater. 14, 117 (2014).

[6] J. Yan, M. Han, J. Zhang, C. Xu, E. Luijten, and S. Granick, Nat. Mater. 15, 1095 (2016).

[7] Y. Suzuki, G. Cardone, D. Restrepo, P. D. Zavattieri, T. S. Baker, and F. A. Tezcan, Nature (London) 533, 369 (2016).

[8] E. Zaccarelli, J. Phys.: Condens. Matter 19, 323101 (2007).

[9] J. M. van Doorn, J. E. Verweij, J. Sprakel, and J. van der Gucht, Phys. Rev. Lett. 120, 208005 (2018).

[10] C. van der Wel, A. Vahid, A. Šarić, T. Idema, D. Heinrich, and D. J. Kraft, Sci. Rep. 6, 32825 (2016).

[11] D. Li, S. Banon, and S. L. Biswal, Soft Matter 6, 4197 (2010).

[12] H. R. Vutukuri, A. F. Demirörs, B. Peng, P. D. J. van Oostrum, A. Imhof, and A. van Blaaderen, Angew. Chem. Int. Ed. 51, 11249 (2012).

[13] J. Palacci, S. Sacanna, A. P. Steinberg, D. J. Pine, and P. M. Chaikin, Science 339, 936 (2013).

[14] G. Meng, N. Arkus, M. P. Brenner, and V. N. Manoharan, Science 327, 560 (2010).

[15] Z. Zeravcic and M. P. Brenner, Proc. Natl. Acad. Sci. USA 111, 1748 (2014).

[16] P. Cicuta and D. Vella, Phys. Rev. Lett. 102, 138302 (2009).

[17] B. D. Leahy, L. Pocivavsek, M. Meron, K. L. Lam, D. Salas, P. J. Viccaro, K. Y. C. Lee, and B. Lin, Phys. Rev. Lett. 105, 058301 (2010).

[18] S. Singamaneni and V. Tsukruk, Soft Matter 6, 5681 (2010).

[19] A. Tordesillas, D. Covey, A. B. Croll, J. Shi, and B. Gurmessa, Granul. Matter 16, 249 (2014).

[20] D. Vella, E. du Pontavice, C. L. Hall, and A. Goriely, Proc. R Soc. A 470, 20130609 (2014).

[21] C. P. Broedersz and F. C. MacKintosh, Rev. Mod. Phys. 86, 995 (2014).
[22] K. Bertoldi, V. Vitelli, J. Christensen, and M. van Hecke, Nat. Rev. Mater. 2, 17066 (2017).

[23] M. Dogterom and B. Yurke, Science 278, 856 (1997).

[24] O. Chaudhuri, S. H. Parekh, and D. A. Fletcher, Nature (London) 445, 295 (2007).

[25] J. Paulose, G. A. Vliegenthart, G. Gompper, and D. R. Nelson, Proc. Natl. Acad. Sci. USA 109, 19551 (2012).

[26] X. Mao, A. Souslov, C. I. Mendoza, and T. C. Lubensky, Nat. Commun. 6, 5968 (2015).

[27] K. Baczynski, R. Lipowsky, and J. Kierfeld, Phys. Rev. E 76, 061914 (2007).

[28] J. Kierfeld, K. Baczynski, P. Gutjahr, T. Kühne, and R. Lipowsky, Soft Matter 6, 5764 (2010).

[29] E. Pilyugina, B. Krajina, A. J. Spakowitz, and J. D. Schieber, Polymers 9, 99 (2017).

[30] T. E. Kodger, R. E. Guerra, and J. Sprakel, Sci. Rep. 5, 14635 (2015).

[31] S. G. Stuij, M. Labbé-Laurent, T. E. Kodger, A. Maciołek, and P. Schall, Soft Matter 13, 5233 (2017).

[32] A. Maciołek and S. Dietrich, Rev. Mod. Phys. 90, 045001 (2018).

[33] D. Allan, T. Caswell, N. Keim, and C. van der Wel, trackpy: Trackpy v0.3.2, 2016, available at https://zenodo.org/record/ 60550\#.XXXB2S3MyYU

[34] D. S. Bedi and X. Mao, Phys. Rev. E 92, 062141 (2015).

[35] J. W. Hutchinson and W. T. Koiter, Appl. Mech. Rev. 23, 1353 (1970).

[36] Z. P. Bazant and L. Cedlin, Stability of Structures (World Scientific, Singapore, 2009).

[37] C. Coulais, J. T. B. Overvelde, L. A. Lubbers, K. Bertoldi, and M. van Hecke, Phys. Rev. Lett. 115, 044301 (2015).

[38] See Supplemental Material at http://link.aps.org/supplemental/ 10.1103/PhysRevResearch.1.023033 for two experimental microscopy videos and two simulation animations.

[39] A. D. Dinsmore, V. Prasad, I. Y. Wong, and D. A. Weitz, Phys. Rev. Lett. 96, 185502 (2006).

[40] J. P. Pantina and E. M. Furst, Phys. Rev. Lett. 94, 138301 (2005). 
[41] S. L. Biswal and A. P. Gast, Phys. Rev. E 68, 021402 (2003).

[42] D. L. Ermak and J. A. McCammon, J. Chem. Phys. 69, 1352 (1978).

[43] A. Magnusson, M. Ristinmaa, and C. Ljung, Int. J. Solids Struct. 38, 8441 (2001).
[44] F. Gittes, B. Mickey, J. Nettleton, and J. Howard, J. Cell Biol. 120, 923 (1993).

[45] A. Tordesillas, J. Zhang, and R. Behringer, Geomech. Geoeng. 4, 2 (2009).

[46] A. Tordesillas, D. M. Walker, and Q. Lin, Phys. Rev. E 81, 011302 (2010) 